\title{
Multibound soliton formation in an erbium- doped ring laser with a highly nonlinear resonator
}

\author{
Dmitriy A. Dvoretskiy, Stanislav G. Sazonkin, Igor S. Kudelin, Ilya O. Orekhov, Alexey B. Pnev, \\ Valeriy E. Karasik, Lev K. Denisov
}

\begin{abstract}
We have studied the generation of low-noise ultrashort multibound solitons in the telecommunication spectral window in an erbium-doped all-fiber ring laser with a highlynonlinear resonator mode-locked by a nonlinear polarization evolution effect. The multibound soliton generation is obtained with more than 20 bound dechirped pulses with a duration of 240 fs at a repetition rate of $\sim 11.3 \mathrm{MHz}$ (with a signal-to-noise ratio of $\sim 73.3 \mathrm{~dB}$ ), the relative intensity noise is $<-140 \mathrm{dBc} / \mathrm{Hz}$, and the Allan deviation of the repetition frequency does not exceed $\sim 1.3 \cdot 10^{-8}$ with a time averaging window of $\sim 100 \mathrm{~s}$.
\end{abstract}

Index Terms-fiber lasers, fiber nonlinear optics, laser mode locking, ultrafast lasers

\section{INTRODUCTION}

$\mathrm{U}$ LTRA-SHORT pulsed (USP) mode-locked (ML) fiber lasers can be considered as an ideal platform to expand future applications due to the complex nonlinear dynamics with a presence of a high value of a group velocity dispersion (GVD) and a third-order dispersion (TOD) in the laser resonator [1]. The multibound solitons (MBSs) generation regime [2], soliton molecules [1] and soliton crystals [3] involve a bound state a bound state of multiple optical pulses that propagate with a fixed temporal separation through optical fibers. MBSs are of considerable interest in various fields, such as telecommunication transmission systems [1], optical metrology for astronomical spectrograph calibration [4], nonlinear photonic signal processing [5], medical applications [6] and coherent pulse staking amplification [7]. Note that most of the mentioned applications require the long-term and low-noise operation of an MBS laser.

Since the first numerical demonstration in Ref. [8] MBS generation has been experimentally obtained by different ML techniques. For example, the highest repetition rate of up to 10 $\mathrm{GHz}$ in fiber lasers can be achieved by active mode locking [9] or even up to $50 \mathrm{GHz}$ in the case of a Kerr comb in a thermally controlled on-chip silica glass microresonator [10]. Passive

The authors would like to thank A.A. Krylov, S.V. Firstov, A. K. Senatorov, M.A. Likhachev (FORC RAS) for the provision of the active fiber and HNLF, GVD measurements, and a fruitful discussion. The reported study was funded by RFBR, according to the research projects Nos 18-32-20017 and 18-3800615 .

Dmitriy A. Dvoretskiy, Stanislav G. Sazonkin, Ilya O. Orekhov, Alexey B Pnev, Valeriy E. Karasik, Lev K. Denisov are with Bauman Moscow State mode-locking techniques include the generation of an MBS by different kinds of real saturable absorbers such as SESAM [11], carbon nanotubes [1], graphene [12], $\mathrm{MoS}_{2}$ and $\mathrm{Sb}_{2} \mathrm{Te}_{3}$ saturable absorbers $[13,14]$. MBS generation has also been widely observed in classic NPE-based fiber lasers [15, 16]. Due to the soliton energy quantization effect theoretically investigated in [17] which leads to multiple pulsing it is possible to generate of several hundred solitons in one packet by pump power scaling, as demonstrated in Ref. [18, 19]. Moreover, harmonic mode locking in a Mach-Zehnder fiber laser enables the tunable generation of multiple pulses at a high-repetitionrate $[20,21]$.

Unfortunately, there are no significant data on the low-noise and reliable long-term performance of the obtained MBS lasers. In this paper, together with [22], we suggest a simple method to achieve the reliable long-term operation of multiple pulses by increasing the nonlinearity of an all-fiber resonator, which leads to the formation of low-noise ultrashort multibound solitons with $\mathrm{N} \sim 26$ bound pulses in an NPE-based ML fiber ring laser.

\section{EXPERIMENTAL SETUP}

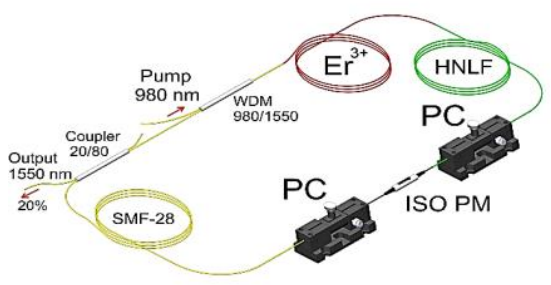

Fig. 1. Experimental setup of the ML EDF ring laser.

The experimental setup of the ML erbium-doped fiber (EDF) ring laser with a highly-nonlinear resonator is shown in Fig. 1. A commercially developed isolator-polarizer (ISO PM) was used as a USP filter for mode locking based on the nonlinear polarization evolution effect and ensured unidirectional generation.

Technical University, Moscow 107005 Russia (e-mail: ddvoretskiy@bmstu.ru).

Igor S. Kudelin is with the Aston Institute of Photonics Technologies, Aston University, Aston triangle, Birmingham, B4 7ET, UK (e-mail: kudelini@aston.ac.uk).

Copyright (c) 2019 IEEE 

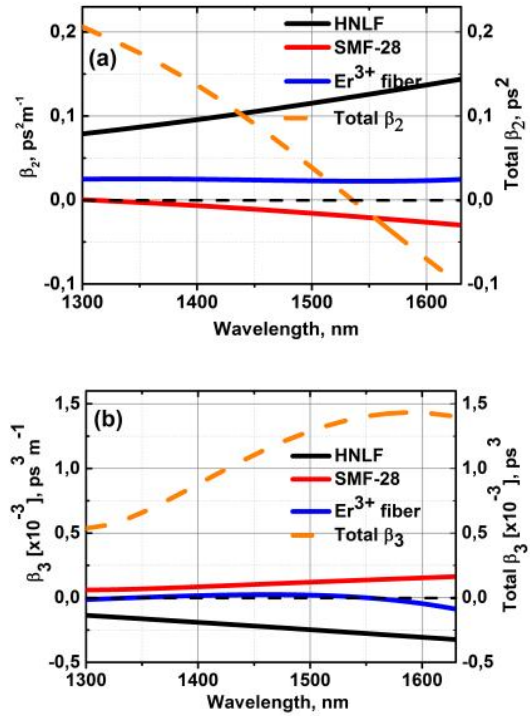

Fig. 2. (a) GVD and $\beta_{2}$ net-cavity dispersion parameter. (b) TOD and $\beta_{3}$ netcavity dispersion parameter for the ML EDF ring laser.

To adjust the ML regime two polarization controllers (PCs) located at both ends of the ISO PM were included in the ring cavity. We used a pigtailed single mode laser diode operating at $980 \mathrm{~nm}$ with a maximum output power of $500 \mathrm{~mW}$ as the pump source for the erbium-doped fiber (EDF). An 80/20 fiber coupler was used to lead the laser radiation out of the cavity. The all-fiber ring cavity was formed by a $3.6 \mathrm{~m}$ active EDF with a low signal core absorption of $\sim 6.5 \mathrm{~dB} / \mathrm{m}$ at the pump wavelength and a dispersion of $\mathrm{D} \sim-17.4 \mathrm{ps} /(\mathrm{nm} \cdot \mathrm{km})$ at 1550 $\mathrm{nm}$ and a highly nonlinear germanosilicate fiber with a $\sim 50$ mol.\% concentration of germanium oxide in the core with a dispersion of $\mathrm{D} \sim-100 \mathrm{ps} /(\mathrm{nm} \cdot \mathrm{km})$ at $1550 \mathrm{~nm}, \sim 11.5 \mathrm{~m} \mathrm{SMF-}$ 28 fiber (Corning Corp.) with a dispersion of D 17.4 $\mathrm{ps} /(\mathrm{nm} \cdot \mathrm{km})$ at $1550 \mathrm{~nm}$ was used to reduce the total net-cavity GVD into a slightly negative region. The HNLF production parameters were the same as those in [23] and the measured core diameter was $\sim 2.5 \mu \mathrm{m}$. The calculated value of the nonlinear refractive index $\mathrm{n}_{2}$ was $3.63 \cdot 10^{-16} \mathrm{~cm}^{2} / \mathrm{W}$. The dispersion parameters $\beta_{2}$ and for the fibers are shown in Fig. 2. The net-cavity dispersion parameters are presented for the HNLF length of $\mathrm{L}_{\mathrm{HNLF}} \sim 1.53 \mathrm{~m}$. Note, that the dispersion parameters $\beta_{2}$ and $\beta_{3}$ have been recalculated from the chromatic dispersion of the fibers measured by the interferometric technique described in [24].

\section{EXPERIMENTAL RESULTS AND DISCUSSION}

Previously we achieved stretched pulse generation in a similar laser resonator with compensated net-cavity GVD and significant third-order dispersion in the highly nonlinear laser resonator [25]. Here we demonstrate multibound soliton formation occurred at the high value of a pump power of $\sim 300$ $\mathrm{mW}$. The output power versus the pump power is shown in Fig. 3 with a maximum output average power of $30 \mathrm{~mW}$. The MBS ML threshold is observed by means of adjusting both PCs at an average pump power of $\sim 300 \mathrm{~mW}$. It should also be pointed out that the control of the PC adjustment at the threshold pump power affected the temporal separation of the ultrashort pulses in the autocorrelation trace and output pulse spectra but did not affect the maximal number of pulses in the bound state. Detailed information about the soliton number evolution in a bunch versus the pump power can be found in our previous paper [26]. Moreover, we did not observe any pulse breaking by increasing the pump power up to $500 \mathrm{~mW}$. The decay of the MBS regime was observed at a pump power of $\sim 130 \mathrm{~mW}$. The

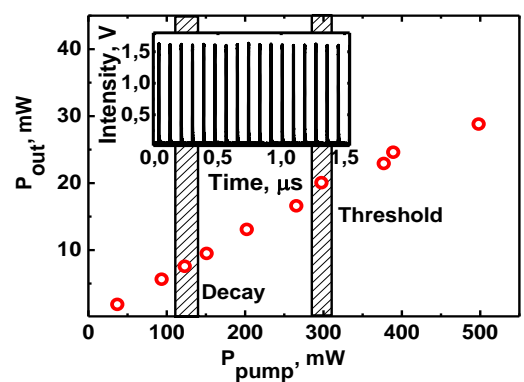

Fig. 3. Pump vs. Output Power. Inset: output pulse trace.

presence of significant hysteresis between the threshold and the decay pump power indicates the generation regime stability, as shown in Ref. [17]. The inset of Fig. 3 shows a typical output pulse trace for the USP generation (using the oscilloscope Infinium MSO9254A; Keysight Technologies, Santa Rosa, CA, USA).

Fig. 4 (a) shows the output multibound soliton generation spectrum in arbitrary units and on a $\mathrm{dB}$ scale (inset) with the spectral dependence of the net-cavity GVD. It should be noted that the MBS generation regime occurred only in the slightly negative region close to zero of the net-cavity GVD and with the presence of an uncompensated TOD (see Fig. 2).
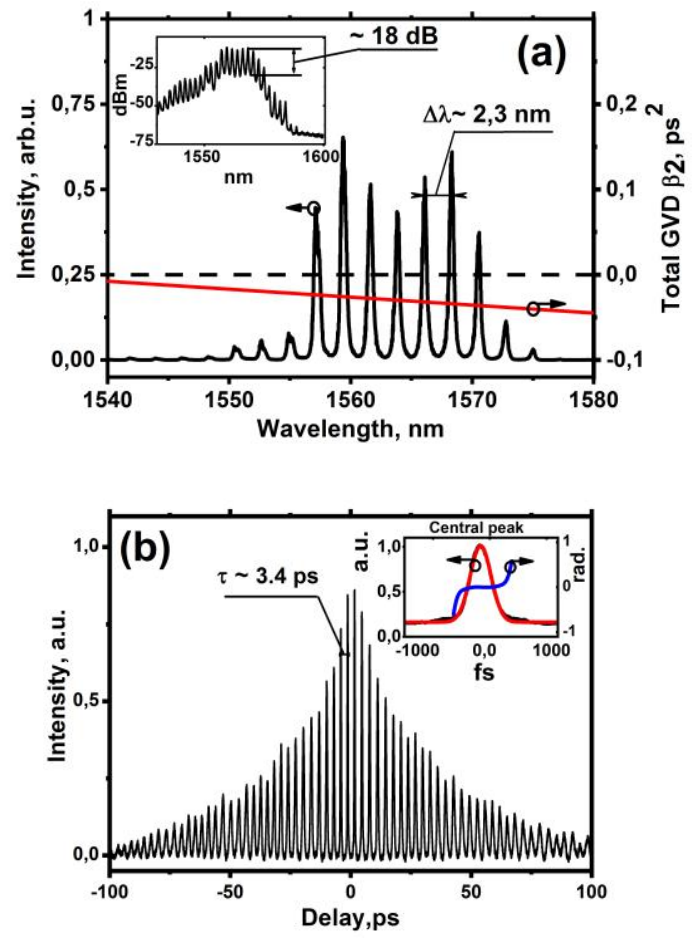

Fig. 4. (a) Pulse spectra and net-cavity GVD. Inset: pulse spectra on a $\mathrm{dB}$ scale. (b) Intensity autocorrelation trace. Inset: the central peak of the auto-correlation trace with Gaussian fitting and the pulse phase. 
The output spectrum (see Fig. 4(a)) evidently shows highcontrast intensity fringes $(\sim 18 \mathrm{~dB})$ that indicate a stable and strong coherent connection between the ultrashort pulses and fixed coherent phase difference in the temporal domain

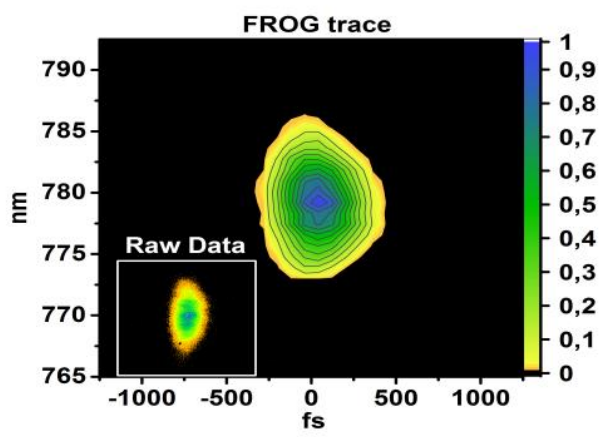

Fig. 5. SHG FROG trace of the MBS generation.

between several identical pulses [1]. The lack of symmetry could be attributed to the influence of high-order dispersion and the phase difference of $\pi / 2$ between bound solitons [27]. The constant period of the sharp intensity fringes indicates the presence of several bound pulses with a stable time separation between them [15].

Fig. 4 (b) shows an intensity autocorrelation trace of the observed MBS generation (obtained by using the autocorrelator FR-103WS; FEMTOCHROME RESEARCH INC., Berkeley, CA, USA) and USP phase (by using Swamp Optics LLC, GRENOUILLE Model 15-40-USB, Georgia, USA) and the central autocorrelation peak with Gaussian fitting and the pulse phase (inset). More than 20 bound pulses are obtained with a constant intertemporal width of $\sim 3.4$ ps and a central peak duration of $<240$ fs at full width at half maximum (FWHM) assuming Gaussian fitting which is $\sim 19$ pulse widths between pulses. The period of the spectral modulation (see Fig. 4(a)) is $\Delta \lambda=2.3 \mathrm{~nm} \pm 0.5 \mathrm{~nm}(\Delta v=281.3 \mathrm{GHz})$. The spectral modulation interfringe is inversely proportional to the temporal separation of the pulses $\tau=1 / \Delta v$. Thus, the calculated bound soliton separation $\tau=3.6 \mathrm{ps} \pm 1.2 \mathrm{ps}$ corresponds well to the experimental observation from the autocorrelation trace (3.4 ps). The Gaussian form of the autocorrelation central peak without a pedestal and the almost symmetrical form of the autocorrelation without additional intensity peaks along with the observed output spectra indicate that one pulse duration in the bond state is $\tau_{\text {pulse }}<240$ fs at FHWM with a stable temporal separation of pulses. Note that the asymmetrical form of the autocorrelation trace connected with the peak intensity can be attributed to the long averaging time of one trace exposure (100 times $x 1 \mathrm{~s} \sim 1.5 \mathrm{~min}$ ) and the significant noise floor of the autocorrelator.

Fig. 5 shows a second-harmonic generation (SHG) frequency-resolved optical gating (FROG) trace of the central autocorrelation peak, and the inset shows raw data from the matrix photodetector of the SHG FROG with a clear band structure. Moreover, we observed dechirped MBS generation according to the clean and symmetrical shape of the FROG trace and recalculated USP phase (inset of Fig. 4(b)). The calculated time-bandwidth product (TBP) for the spectral pulse width $\Delta \lambda_{\mathrm{FWHM}} \sim 15 \mathrm{~nm}\left(\Delta v_{\mathrm{FWHM}}=1.86 \mathrm{THz}\right.$, see Fig. $\left.4(\mathrm{a})\right)$ and the minimal pulse width $\tau_{\min } 235$ fs of the MBS is TBP $=$ $\Delta v \times \tau_{\min } \approx 0.446$ (the typical TBP value for Gaussian pulses is $\approx$ 0.441 ). Thus, the obtained MBS is close to its bandwidth-limit and dechirped character of the MBS generation is confirmed.

To characterize the short-term stability of the obtained MBS generation we measured the radio-frequency (RF) spectrum of the output pulse train and the relative intensity noise (RIN) of the developed laser at the maximal pump power of $500 \mathrm{~mW}$. Fig. 6 shows a typical RF spectrum for the MBS regime at the fundamental oscillator frequency with a resolution of $300 \mathrm{~Hz}$ (using the ESA FSL 3 model.03; Rohde \& Schwarz GmbH \&

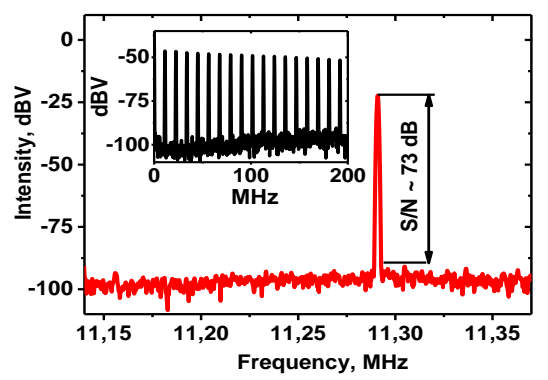

Fig. 6. RF spectrum of the pulse train at the repetition frequency. Inset: RF spectrum in the range $0-200 \mathrm{MHz}$.

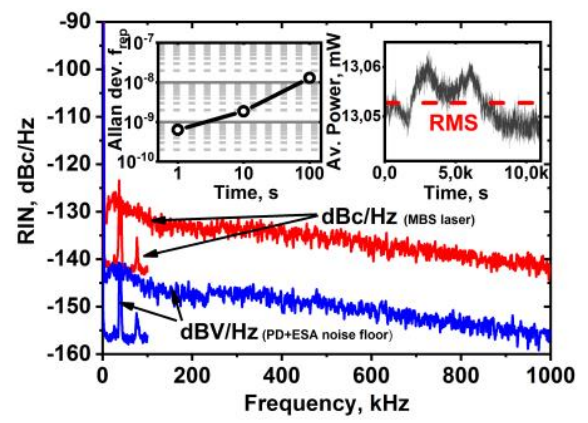

Fig. 7. RIN of the ML laser and noise floor from the PD+ESA. Inset: Allan deviation of the repetition rate and average output optical power during the measurement.

Co. KG, Munich, Germany). The RF spectrum has a peak at a frequency of $\sim 11.3 \mathrm{MHz}$ with a signal-to-noise (SNR) ratio of $\sim 73 \mathrm{~dB}$, and the absence of sidebands indicates the pulse-topulse MBS energy stability. The inset of Fig. 6 shows the RF spectrum in the frequency range of $30 \mathrm{kHz}-200 \mathrm{MHz}$ (resolution bandwidth of $3 \mathrm{kHz}$ ). The high SNR at the fundamental frequency and the absence of Q-switched sidebands in the RF spectrum prove the pulse-to-pulse stability of the ML regime.

Fig. 7 shows the RIN of the MBS laser (in $[\mathrm{dBc} / \mathrm{Hz}]$ ) at the maximal pump power of $500 \mathrm{~mW}$ and the noise floor from the photodetector (PD) and the electrical spectrum analyzer (in [dBV/Hz]) over a frequency range of $30 \mathrm{~Hz}-1000 \mathrm{kHz}$ (using ESA SR770FFT, Stanford Research Systems, California, USA over the frequency range from $30 \mathrm{~Hz}-100 \mathrm{kHz}$ and the Rohde \& Schwarz ESA, above $100 \mathrm{kHz}$ ). For the MBS pulses at frequencies of $\sim 30 \mathrm{~Hz}-100 \mathrm{kHz}$, the RIN value was below $-140 \mathrm{dBc} / \mathrm{Hz}$, while at frequencies up to $1000 \mathrm{kHz}$, the RIN 
value was below $-125 \mathrm{dBc} / \mathrm{Hz}$. Thus, we obtained an $\mathrm{RIN}$ value for the MBS laser that is comparable to that obtained previously by femtosecond combs based on other ML principles and USP generation regimes [28].

The long-term stability of the MBS generation can be characterized by the Allan deviation of the repetition rate (see the inset of Fig. 7 with $5 \mathrm{~Hz}$ data using Universal Counter 53132A, Agilent Inc., Santa Clara, CA, USA) for the freerunning MBS fiber laser. The developed MBS laser has a relatively low repetition rate deviation with the value of $6.2 \cdot 10^{-10}$ for a $1 \mathrm{~s}$ interval, which is near the limit of the quartz oscillator frequency uncertainty and is determined only by the temperature drift during the averaging time interval of $1-1 \cdot 10^{2} \mathrm{~s}$. It should be noted that we did not observe any pulse breaking or operation instabilities of the MBS generation during the experiment that lasted several hours. The inset of Fig. 7 demonstrates the average optical output power stability with a standard deviation of $\sim 0.03 \%$ RMS over a time of $10^{4} \mathrm{~s}$ (measured by PM200 power meter with InGaAs detector S145C, Thorlabs Inc., Newton, New Jersey, US).

We obtained lower noise in the MBS operation at a high pump power than the previously observed high-energy ultrashort stretch-pulse generation in the same NPE-based ML fiber laser [25]. This fact is in good agreement with the theoretical analysis of the soliton energy quantization effect in a soliton fiber ring laser passively mode-locked by the NPE effect in Ref. [29]. The highest energy of a fundamental soliton with duration $\tau_{\mathrm{p}}$ is limited by the soliton area theorem Es $\left|\beta_{2}\right| /\left(\gamma \cdot \tau_{\mathrm{p}}\right)$, where $\gamma$ is the net nonlinear coefficient and $\beta_{2}$ is the total cavity dispersion. Soliton energy quantization results in pulse splitting at operation powers higher than the fundamental limit. Moreover, the energy quantization effect has been recently experimentally demonstrated by the dispersive Fourier transform technique (or time-stretch technique) in an SESAM mode-locked MBS fiber laser [11] with good agreement with the present results. Thus, MBS generation with $\mathrm{N} \sim 26$ bound states is a natural consequence of the gain competition between multiple solitons and high-energy ultrashort stretch-pulse generation occurs in an NPE-based all-fiber erbium-doped ring laser with highly nonlinear resonator.

\section{CONCLUSIONS}

In conclusion, low-noise multibound soliton generation is obtained (more than 20 bound pulses with an intertemporal width of $\sim 3.4 \mathrm{ps}$ ) with an duration of $\sim 240$ fs at the repetition frequency of $\sim 11.3 \mathrm{MHz}$ (with a signal-to-noise ratio of $\sim 73.3$ $\mathrm{dB}$ ) in the telecommunication spectral window in an erbiumdoped all-fiber ring laser with a highly-nonlinear resonator mode locked by an NPE effect. Note that the relative intensity noise is $<-140 \mathrm{dBc} / \mathrm{Hz}$, and the Allan deviation of the repetition frequency does not exceed $\sim 1.3 \cdot 10^{-8}$ with a time averaging window of $\sim 100 \mathrm{~s}$.

\section{REFERENCES}

[1] Chernysheva, M. et al., "Double-Wall Carbon Nanotube Hybrid ModeLocker in Tm doped Fibre Laser: A Novel Mechanism for Robust BoundState Solitons Generation,”Sci. Rep., vol. 7, p. 44314, March 2017.
[2] Nhan Duc Nguyen, Le Nguyen Binh, "Generation of high order multibound solitons and propagation in optical fibers," Optics Communications, vol. 282, no. 12, pp. 2394-2406, 2009.

[3] A. Haboucha et. al., "Analysis of soliton pattern formation in passively mode-locked fiber lasers," Phys. Rev. A., vol. 78, p. 043806, 2008.

[4] Zajnulina et. al., "Characteristics and stability of soliton crystals in optical fibres for the purpose of optical frequency comb generation," Optics Communications, vol. 393, pp. 95-102, 2017.

[5] Le Nguyen Binh, Optical Multi-Bound Solitons. Optics and Photonics., Taylor \& Francis, 2015.

[6] Can Kerse et al., "Ablation-cooled material removal with ultrafast bursts of pulses," Nature, vol. 537, pp. 84-88, 2016.

[7] Tong Zhou et al., "Coherent pulse stacking amplification using lowfinesse Gires-Tournois interferometers," Opt. Express, vol. 23, pp. 74427462, 2015.

[8] Boris A. Malomed, "Bound solitons in coupled nonlinear Schrödinger equations," Phys. Rev. A, vol. 45, p. R8321, 1992.

[9] C. Luo, S. Wang, and Y. Lai, "10 GHz Bound Soliton Mode-locking in an Environmentally Stable FM Mode-locked Er-doped Fiber Soliton Laser," in CLEO: 2014, OSA Technical Digest (online) (Optical Society of America, 2014), p. JTu4A.69, 2014.

[10] Weiqiang Wang et al., "Robust soliton crystals in a thermally controlled microresonator," Opt. Lett., vol. 43, pp. 2002-2005, 2018.

[11] Shuqian Sun et al., "Time-stretch probing of ultra-fast soliton dynamics related to Q-switched instabilities in mode-locked fiber laser," Opt. Express, vol. 26, pp. 20888-20901, 2018.

[12] Y. F. Song et al., "Coexistence and interaction of vector and bound vector solitons in a dispersion-managed fiber laser mode locked by graphene," Opt. Express, vol. 24, pp. 1814-1822, 2016.

[13] Yadong Wang et al., "Harmonic mode locking of bound-state solitons fiber laser based on $\mathrm{MoS}_{2}$ saturable absorber," Opt. Express, vol. 23, pp. 205-210, 2015.

[14] Zhenhong Wang et al., "Generation of harmonic mode-locking of bound solitons in the ultrafast fiber laser with $\mathrm{Sb}_{2} \mathrm{Te}_{3}$ saturable absorber on microfiber," Laser Physics Letters, vol. 16, p. 025103, 2019.

[15] Ph. Grelu et al., "Phase-locked soliton pairs in a stretched-pulse fiber laser," Opt. Lett., vol. 27, pp. 966-968, 2002.

[16] L. M. Zhao et al., "Bound states of dispersion-managed solitons in a fiber laser at near zero dispersion," Appl. Opt., vol. 46, pp. 4768-4773, 2007.

[17] A. Komarov et al., "Multistability and hysteresis phenomena in passively mode-locked fiber lasers," Phys. Rev. A, vol. 71, p. 053809, 2005.

[18] Adil Haboucha et al., "Coherent soliton pattern formation in a fiber laser," Opt. Lett., vol .33, pp. 524-526, 2008.

[19] Foued Amrani et al.,"Universal soliton pattern formations in passively mode-locked fiber lasers," Opt. Lett., vol. 36,pp. 1545-1547, 2011.

[20] Jérôme Lhermite et al.,"Tunable high-repetition-rate fiber laser for the generation of pulse trains and packets," Opt. Lett., vol. 32, p. 1734, 2007.

[21] A. Andrianov and A. Kim, "Extremely elastic soliton crystals generated in a passively mode-locked tunable high-repetition-rate fiber laser," arXiv [physics.optics], 1905.03129v1, 2019.

[22] D. A. Dvoretskiy et al., "Ultrashort Multi-Bound Solitons Generation in the Passively Mode-Locked All-Fiber Laser at the Telecom Window," 2018 European Conference on Optical Communication (ECOC), Rome, 2018, pp. 1-3.

[23] Y. Yatsenko and A. Mavritsky, "D-scan measurement of nonlinear refractive index in fibers heavily doped with $\mathrm{GeO} 2$," Opt. Lett., vol. 32, pp. 3257-3259, 2007.

[24] A.E. Levchenko, et al., "Measurement of dispersion in optical fibres with a microstructure cladding," Quantum Electronics, vol. 35, no 9, pp. 835$838,2005$.

[25] Dmitriy A. Dvoretskiy et al., "High-energy ultrashort-pulse all-fiber erbium-doped ring laser with improved free-running performance," $J$. Opt. Soc. Am. B, vol. 35, pp. 2010-2014, 2018.

[26] Dmitriy A. Dvoretskiy et al., "Multibound solitons generation with a controllable number of bound states in a passive mode-locked all-fiber erbium-doped ring laser," Proc. SPIE, vol. 11026, p. 110260Q, 2019.

[27] Lili Gui et al., "Soliton Molecules and Multisoliton States in Ultrafast Fibre Lasers: Intrinsic Complexes in Dissipative Systems," Applied Sciences, vol. 8, no. 2, p. 201, 2018.

[28] Jungwon Kim and Youjian Song, "Ultralow-noise mode-locked fiber lasers and frequency combs: principles, status, and applications," Adv. Opt. Photon., vol. 8, pp. 465-540, 2016.

[29] D. Y. Tang, L. M. Zhao, B. Zhao, and A. Q. Liu "Mechanism of multisoliton formation and soliton energy quantization in passively modelocked fiber lasers," Phys. Rev. A, vol. 72, p. 043816, 2005. 